\title{
Phase trajectories of non-linear oscillations of a tower structure with an attached damper in a uniform wind flow
}

\author{
Viktorija Volkova ${ }^{1}$, Leonids Pakrastinsh ${ }^{2}$, Liga Gaile ${ }^{3}$ \\ ${ }^{I}$ Department of Construction, Geotechnics and Geomechanics, Dnipro University of Technology, Dnipro, Ukraine \\ ${ }^{2,3}$ Faculty of Civil Engineering, Riga Technical University, Riga, Latvija \\ E-mail: 1drvev09@gmail.com (corresponding author)
}

\begin{abstract}
This paper is devoted to the study of the dynamic behaviour of a tower structure with an attached damper. Two dampers are attached to the upper end of the rod in two mutually perpendicular planes. The oscillation damper is a mass attached to the rod by elastic coupling and a viscous friction. The mathematical model of the object being under consideration includes a system of two non-linear differential equations. The numerical simulation was used to study the dynamic behavior of a dissipative system with limited excitation, containing a pendulum vibration damper. The characteristics of the system amplitude - the speed of the wind flow is obtained. It is shown that the pendulum vibration damper can significantly reduce the amplitudes of resonant oscillations of the cantilever rod. It is also shown that the choice of system parameters makes it possible to avoid the establishing of resonant oscillations of the system. at the first resonance zone where the amplitudes of oscillations achieved the greatest values.
\end{abstract}

Keywords: tower, pendulum damper, wind flow, numerical simulation, dynamic behavior.

\section{Introduction}

The phenomena observed in the interaction of structures and their elements with the wind flow are very diverse. The most famous are wind resonance, galloping and buffing. They are very complex and not all of them remain poorly defined. Traditionally, the wind effect is assumed to be a combination of the average steady-state pressure and random pulsations caused by the turbulence of the wind flow. Such an idealization of interaction between the structure and the wind flow allows to predict and investigate the main aerodynamic effects and does not cause additional computational difficulties (Korenev, 1984).

Steady-state wind pressure in addition to the static effect can lead to the establishment of long-term structureoscillations in a wide range of wind speed and under the certain conditions. This phenomenon was often observed in the nature and had been called as a wind resonance. Such oscillations usually occur in a plane, which is perpendicular to the flow direction. Previously it was experimentally established that it represents a self-oscillatory process caused by the breakdown of the vortices at the extreme points. Pulsations of the wind flow lead to the appearance of random oscillations in the plane of the wind flow. The amplitude of self-oscillations significantly depends on the damping value. Therefore, the application of dampers is a very effective way to reduce the level of oscillations.

\section{Damper absorbers}

The design and theory of linear oscillation dampers are well known and widely represented in the references (Kauderer, 1961; Z. A. Shulman \& I. Z. Shulman, 2013). Unfortunately, such devices are used for reduction of the amplitudes of forced oscillations only in a narrow frequency range. Non-linear oscillation dampers are effective in a larger frequency range (Mikhlin \& Reshetnikova, 2005). The operation of the system with such a damper is considered in research/publication of (Avramov \& Mikhlin, 2006). Its various designs are also presented there. The oscillation damper with one degree of freedom was proposed in several publications (Avramov \& Mikhlin, 2005; Cuvalci \& Ertas, 1996) for damping of the forced oscillations. In (Natsiavas, 1992), it was shown that a non-linear damper reduces the amplitudes of oscillations in a wide frequency range of the excitation force. Energy transfer processes to a substantially non-linear absorber are studied in (Gendelman, 2004). A handful of American laboratories have proposed the application of non-linearity for the absorption of oscillations (Vakakis \& Gendelman, 2001; Viguie \& Kerschen, 2012). As it is shown in research/study (Kerschen, Kowtko, McFarland, Bergman, \& Vakakis, 2007), the non-linear energy sink, an essentially nonlinear absorber, can extract energy from any mode of a host structure. 
The efficiency of oscillation dampers depends on the ratio of its mass $m$ and the value of $M$, hereinafter referred as the main mass. It increases, as a rule, when the damper is installed at a point with the maximum ordinate of the corresponding oscillation mode of the structure.

It should be noted that the application of the pendulum type dampers has a number of limitations. Thus, with an increase in the natural oscillation periods of high-rise structures the use of dynamic oscillation dampers in the form of an ordinary pendulum becomes unacceptable due to a significant increase of the length of its suspension. Oscillation dampers of such sizes cannot be implemented in practice. Therefore, it is necessary to develop other structural design for dynamic oscillation dampers. One of such fundamentally new designs is an oscillation damper in the form of an inverted pendulum.

The effect of damper to the oscillations of the structure on higher modes can be neglected because its frequencies are remote from the partial frequency of oscillation dampers. Thus, it can be assumed to be the same as in the absence of an oscillation damper. Although non-linear oscillation dampers can absorb disturbances in wide range of frequencies due to their increased bandwidth, their performance critically depends on the motion amplitude (Viguie \& Kerschen, 2012). The linear and non-linear dampers general theory is presented in the handbook (Frolov, 1995).

\section{Governing equations of oscillations of the tower structure with an attached damper in a uniform wind flow}

The structural scheme of the tower structure is considered as a cantilever rod having a variable cross section. The base of the rod is rigidly clamped. Two dampers are attached to the upper end of the rod in two mutually perpendicular planes. The oscillations damper is a mass attached to the rod by elastic coupling and a viscous friction.

The mathematical model of the research object includes a system of two non-linear differential equations. To simplify the analysis of the studied dynamic system, the internal inelastic resistance of the rod was taken into account according to the Voigt hypothesis. This assumption is quite true in view of the smallness of the value of internal resistance with respect to the values of the aerodynamic force and the viscous resistance of the damper.

Rod motions with a pendulum damper attached in a uniform wind flow are described by a system of partial differential equations

$$
\left\{\begin{array}{l}
\left(1+\gamma_{0} \frac{\partial}{\partial t}\right)\left[\frac{\partial^{2}}{\partial x^{2}}\left(E I_{x} \frac{\partial^{2} \omega}{\partial x^{2}}\right)\right]+m_{x} \frac{\partial^{2} \omega}{\partial t^{2}}-q_{x}+ \\
+\delta(x-l) m_{1} \frac{\partial^{2}\left(y+\omega_{l}\right)}{\partial t^{2}}=0 \\
\frac{\partial^{2}\left(y+\omega_{l}\right)}{\partial t^{2}}+\mu \frac{\partial y}{\partial t}+f_{0}^{2} y=0
\end{array},\right.
$$

where: $m_{x}, E I_{x}, \gamma_{0}$ - the mass of the rod per length, bending stiffness and coefficient of viscous resistance of the rod; $m_{1}, \mu, f_{0}$ - mass, coefficient of viscous resistance and partial frequency of oscillations of a pendulum damper; $\omega, \omega_{l}$ - movement of the rod in an arbitrary cross section $x$ and in section $x=l, y$ - relative movement of the pendulum damper, equal to the elongation of its elastic connection; $q_{x}$ - aerodynamic force acting on the rod; $\delta(x-l)-$ Dirac delta function. wind flow

The value of the aerodynamic force included in the system of Eqs (1) non-linearly depends on the speed of the

$$
q_{x}=0.5 C_{x} \rho h_{x} V_{x}^{2}
$$

where: $\rho$ - the air density; $h_{x}$ - the transverse size of the rod; $V_{x}$ - the value of the velocity of the wind flow, depending on the coordinate cross section of the $\operatorname{rod} x$. The value of the coefficient $C_{x}$ according to the experimental data depends on the shape of the cross section of the rod and can be represented as a polynomial.

$$
C_{x}=\sum_{j=1,3 \ldots}(-1)^{(j-1) / 2} A_{j}\left(\frac{\omega}{V_{x}}\right)^{j} .
$$

In expression (3), the value $A_{j}$ characterizes the cross-sectional shape of the rod. 
Let's limited to two members of the polynomial (3). Thereby from the Eq. (2) we get:

$$
q_{x}=-\left[A_{1}\left(\frac{\omega}{V_{x}}\right)+A_{3}\left(\frac{\omega}{V_{x}}\right)^{3}\right] \rho h_{x} V_{x}^{2}=-A_{1} \omega \rho h_{x} V_{x}+A_{3} \frac{\omega^{3}}{V_{x}} \rho h_{x} .
$$

The frequency and form of structure oscillations are close to the frequency and form of the fundamental tone when tower structures are galloping. Therefore, study of the conditions of the occurrence of self-oscillations is a topic of great interest. Let's assume that:

$$
\omega=U_{x} \lambda(t)
$$

where $U_{x}$ is the first form of oscillations, $\lambda(t)$ is an unknown function of time. For the sake of convenience of further calculations, we will perform the rationing of the first mode of oscillations so that the displacements at the upper end of the rod are equal $U_{l}=1$ and the distribution of the velocity of the wind flow along the height $V_{x}=V v_{x}$ of the rod is represented in the form, where $v_{l}=1, V$ is the flow velocity in the cross section $l$.

Applying the Bubnov-Galerkin procedure to the system of differential Eqs (1) and taking into account (5), we obtain the system of ordinary differential equations:

$$
\begin{gathered}
(1+v) \ddot{\lambda}+\gamma_{0} \omega_{0}^{2} \dot{\lambda}+\omega_{0}^{2} \lambda+v \ddot{y}-0,5 \rho\left(\dot{\lambda} V A_{1} c_{1}-\frac{\dot{\lambda}^{3}}{V} A_{3} c_{3}\right)=0 ; \\
\ddot{\lambda}+\ddot{y}+\mu_{0} \dot{y}+f_{0}^{2} y=0,
\end{gathered}
$$

where $\omega_{0}$ is the frequency of the fundamental tone of the oscillations of the rod, $v$ is the relative mass of the damper, which is equal to:

$$
\mathrm{v}=\frac{m_{1}}{\int_{0}^{l} m_{x} U_{x}^{2} d x}
$$

The values of the coefficients $c_{1}$ and $c_{3}$ are determined by the formulas:

$$
c_{j}=\frac{\int_{0}^{l} U_{x}^{j+1} v_{x}^{2-j} h_{x} d x}{\int_{0}^{l} m_{x} U_{x}^{2} d x} .
$$

The friction force depends on the velocity and does not depend on the coordinate of many real-world systems. The nature of this dependence is determined by the conditions in which the movement takes place. In the general case, the friction force is described by the expression. Note that the magnitude of the friction force vanishes at, i.e. in the absence of movement $\dot{\lambda}=0$. A trait of the studied system is that the friction forces are negative work in some intervals of motion, and positive on others.

\section{Phase trajectories of oscillations of nonlinear systems in the expanded phase space}

Dynamic behaviour of mechanical systems is usually presented as oscillating processes in various graphic forms such as time processes, the Lissajous patterns and hodograph. Such patterns of presentations enable to determine the type of a process and to perform numerical estimations of its characteristics, but do not disclose any properties of the governing system. Unlike them classic phase trajectories have several advantages. A phase space in classic mechanics is represented as a multidimensional space. The number of measured values for a phase space is equal to the doubled number of degrees of freedom of the system being investigated (Andronov, Leontovich, Gordon, \& Mayer, 1966). The state of the system is presented as a point in the phase space, and any change in the system state in time is depicted as the displacement of the point along a line called a phase trajectory. The image of phase plane $(y, \dot{y})$ is a more vivid presentation because it particularly well depicts the inharmonious oscillations. 
Other selection of parameters of phase planes is also possible. A phase trajectory of a plane $(\ddot{y}, y)$ creates even greater interest. The chart $(\dot{y}, \ddot{y})$ can be used for the analysis of dissipative properties of a system, excepting the phase diagram $(\ddot{y}, y)$.

The basic difficulty of construction of the phase diagrams $(\ddot{y}, y)$ and $(\dot{y}, \ddot{y})$ necessity of exclusion of the time dependence $t$ from the corresponding relations. Analytical fulfillment of this operation is not always possible. Preponderance of devices register time-dependent changes of displacements, velocities and accelerations of points of systems under investigation. The sanitary and technological norms determines the limitations of values of mentioned parameters. It is possible to form the related phase diagrams by obtaining the sequentially related couples of parameter values $\ddot{y}(t)$ vs. $y(t)$ or $\ddot{y}(t)$ vs. $\dot{y}(t)$.

As it is shown by the several authors (Volkova \& Schneider, 2005; Volkova, 2013) the expansion of a phase space considering the phase planes $(y, \ddot{y})$ and $(\dot{y}, \ddot{y})$, substantially promotes the efficient analysis of a dynamic system behaviour.

\section{Numerical simulations of dynamic behaviour of tower in uniform wind flow}

For determination of the dependence of the oscillation amplitude of the rod on the speed of the wind flow, a numerical integration of the system of differential Eqs (6-7) was performed.

The amplitudes of oscillations were estimated for 8192 points of the time process. The length of the time histories was 200 cycles of oscillations. Given the possibility of the existence of several stable modes of oscillations in the region of the main resonance, the integration was carried out in two directions - an increase in the velocity of the wind flow - a "forward" pass, and a decrease in speed - a "reverse" pass.

For the sake of convenience of investigations, the normalization of the values of the parameters of the Eqs (6-7) included in the original system was performed. Numerical analysis was performed for the following dimensionless values of system parameters: $v=0.01 ; \omega_{0}=1 ; \gamma_{0}=0.003 ; \rho=1.2 ; c_{1}=0.3 ; c_{3}=0.6 ; A_{1}=1$; $A_{3}=1 ; \mu=0.01 ; f_{0}^{2}=0.5 ; 1 ; 3$. The speed of the wind flow varied within the mentioned limits $V=0,1 \ldots 10$ with increments $\Delta V=0.1$.

First, the technique of reducing was applied for a detailed study of the behaviour of the system at resonance. The results of numerical simulation are shown in Figure 1.

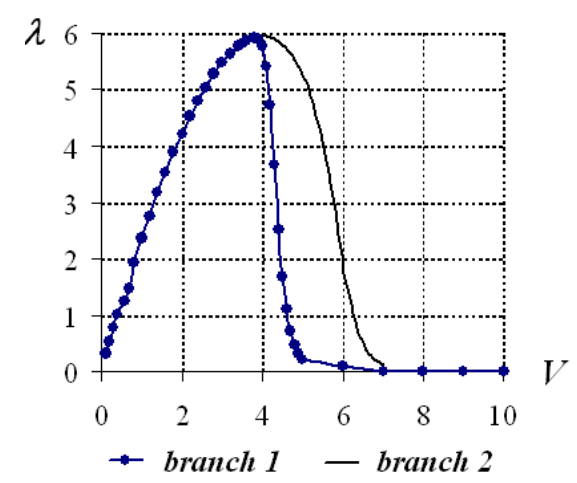

Figure 1. The dependence of the oscillation amplitude of the cantilever rod on the speed of the wind flow in the absence of a pendulum oscillation damper

Branch 1 corresponds to an increase of the wind flow speed, and branch 2 to a decrease. With an increase in the speed of the wind flow the oscillation amplitudes increase and reach a maximum value at a critical value of the speed of the wind flow $V=4.6$. On time processes of this range, a "buildup" of the system is observed (see Figure 2). With a further increase in the speed of the wind flow the oscillation amplitudes decrease, a transition to a non-resonant branch occurs.

During transient time processes, there is a "breakdown" of the amplitudes of the system oscillations. Analyzing the behaviour of branch 2, it can be noted that the establishment of resonant oscillations occurs at the value of the velocity of the wind flow $V=7$. A distinctive feature of the system under study is the presence of a zone of ambiguity of oscillation amplitudes in the range of wind flow velocities $V=4,6 \ldots 7$. The establishment of resonant modes of oscillations in this range is determined solely by the initial conditions. 

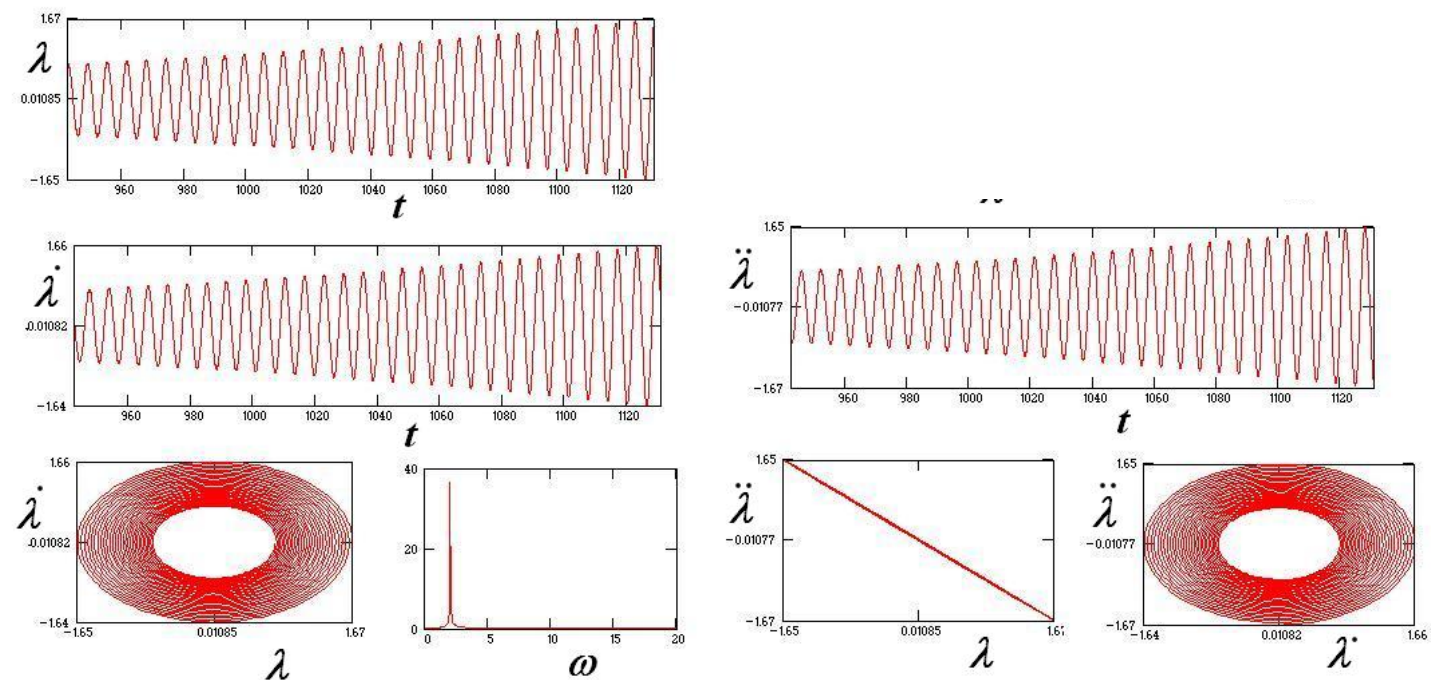

Figure 2. Time histories, spectral characteristics and phase trajectories in expanded space of tower in uniform wind flow

At the second stage, time histories, spectral characteristics and phase trajectories in transient and steady modes of oscillations in a wide range of changes in flow velocity are obtained. Hysteresis effects are found in the resonance region and the zone of interaction of free and forced oscillations (see Figure 3). As we can see in Figure 3, the pendulum vibration damper can significantly reduce the amplitudes of the resonant oscillations of the cantilever rod. It is also shown that the choice of the system parameters makes it possible to avoid establishing the resonant oscillations of the system in the first resonance, where the amplitudes of oscillations are the greatest.
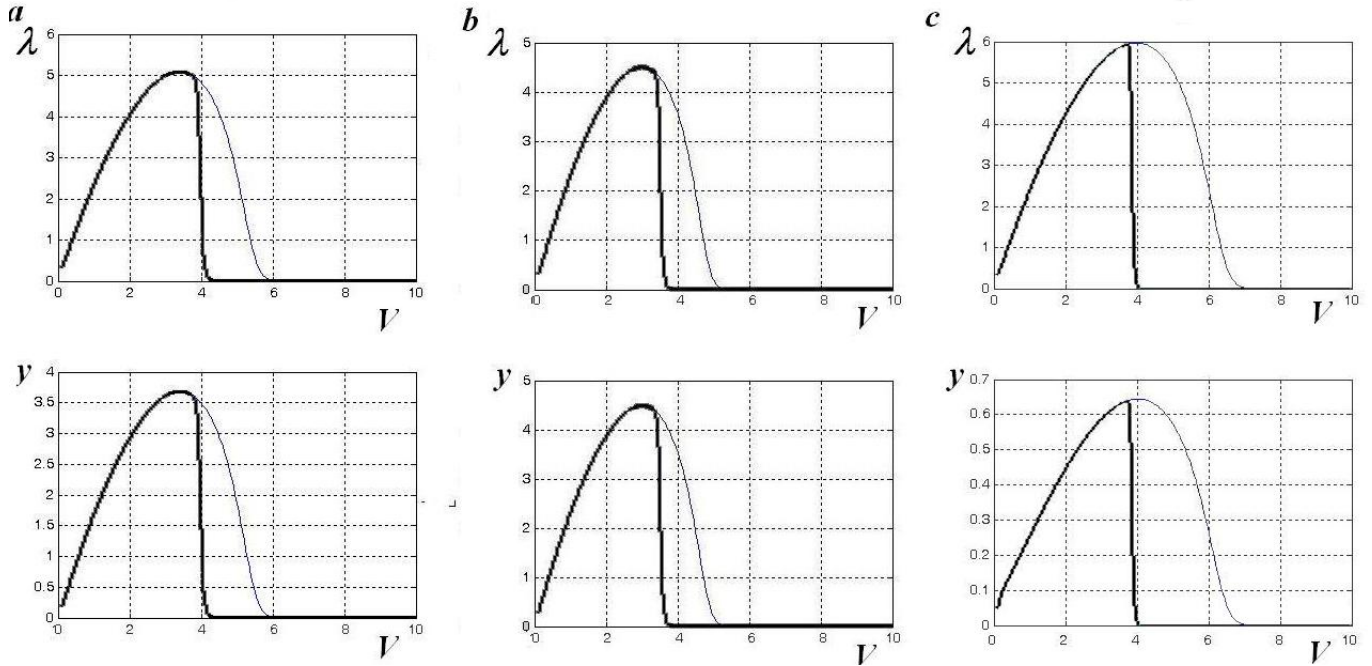

Figure 3. The dependence of the oscillation amplitude of the cantilever rod with attached pendulum damper on the speed

of the wind flow a) $f_{0}^{2}=0.5$; b) $f_{0}^{2}=0.5$; c) $f_{0}^{2}=10$

As we can see in Figure 3, the pendulum vibration damper can significantly reduce the amplitudes of the resonant oscillations of the cantilever rod. It is also shown that the choice of the system parameters makes it possible to avoid establishing the resonant oscillations of the system in the first resonance, where the amplitudes of oscillations are the greatest.

By analyzing the obtained results, one can conclude that it is expedient to use the pendulum dampers with the frequencies of the natural oscillations less than or equal to the frequency of the natural oscillations of the tower $f_{0}^{2} \leq \omega_{0}^{2}$ (Figure 3a, b). The greatest effect of reducing the amplitude of oscillations is achieved at $f_{0}^{2}=\omega_{0}^{2}$. Here is the process of pumping the energy of the oscillations of the tower to the damper. The amplitude of the oscillations of the damper oscillations reached the maximum values and the amplitudes of the oscillations of the tower were re- 
duced. At the same time, the width of the zone of resonance oscillations decreased. Also, the range of values of the wind speed corresponding to the multiplicity of the amplitudes decreased. The application of dampers having frequencies of the natural oscillations greater than the frequency of the own oscillations of the tower does not lead to a significant decrease in the energy of the oscillations of the tower in the wind flow (Figure $3 \mathrm{c}$ ).

\section{Conclusions}

The article investigates the dynamic behaviour of the tower in a uniform wind flow both in the absence of dampers and in the presence of dampers. Time processes and spectral characteristics were obtained by means of the numerical modeling. The article demonstrated that the phenomena inherent in non-linear systems with one degree of freedom remain for systems with two degrees of freedom. At the same time, there are new additional patterns of phase trajectories with a more complex structure appearing in systems with two degrees of freedom. The evaluation of the influence of the initial conditions on the steady-state oscillations was also performed. It is shown that the initial conditions have a significant impact on the dynamics of the studied system. For the first time phase trajectories were obtained in the extended for a nonlinear system with two degrees of freedom. The article demonstrated that the phenomena inherent in nonlinear systems with one degree of freedom remains for systems with two degrees of freedom. At the same time, in systems with two degrees of freedom, new additional patterns of phase trajectories are having a more complex structure.

\section{References}

Andronov, A. A., Leontovich, E. A., Gordon, I. I., \& Mayer, A. G. (1966). The qualitative theory of dynamic systems of the second order. Moscow: State publishing house of the physical and mathematical literature.

Avramov, K. V., \& Mikhlin, Yu. V. (2005). Damping of free elastic vibrations in linear systems. International Applied Mechanics, 4l(2), 203-209. https://doi.org/10.1007/s10778-005-0078-4

Avramov, K. V., \& Mikhlin, Yu. V. (2006). Snap-through truss as an absorber of forced oscillations. Journal of Sound and Vibration, 290, 705-722. https://doi.org/10.1016/j.jsv.2005.04.022

Cuvalci, O., \& Ertas, A. (1996). Pendulum as vibration absorber for flexible structures: experiments and theory. Journal of Vibrations and Acoustics, 118(4), 558-566. https://doi.org/10.1115/1.2888335

Frolov, K. V. (1995). Vibrations in engineering. Moscow: Mashinostroenie.

Gendelman, O. V. (2004). Bifurcations of nonlinear normal modes of linear oscillator with strongly nonlinear damped attachment. Nonlinear Dynamics, 37(2), 115-128. https://doi.org/10.1023/B:NODY.0000042911.49430.25

Kauderer, G. (1961). Non-linear mechanics. Moscow: Publishing house of foreign literature.

Kerschen, G., Kowtko, J. J., McFarland, D. M., Bergman, L. A., \& Vakakis, A. F. (2007). Theoretical and experimental study of multimodal targeted energy transfer in a system of coupled oscillators. Nonlinear Dynamics, 47(1-3), 285-309. https://doi.org/10.1007/s11071-006-9073-5

Korenev, B. G. (1984). Dynamic calculation of buildings and structures. Moscow: Strojizdat.

Mikhlin, Yu. V., \& Reshetnikova, S. N. (2005). Dynamical interaction of an elastic system and essentially nonlinear absorber. Journal of Sound and Vibration, 283(1-2), 91-120. https://doi.org/10.1016/j.jsv.2004.03.061

Natsiavas, S. (1992). Steady state oscillations and stability of non-linear dynamic vibration absorbers. Journal Sound and Vibration, 156(2), 227-245. https://doi.org/10.1016/0022-460X(92)90695-T

Shulman, Z. A., \& Shulman, I. Z. (2013). Testing and monitoring of engineering structures. Dnepropetrovsk: Lira.

Vakakis, A. F., \& Gendelman, O. V. (2001). Energy pumping in nonlinear oscillators: Part II - resonance capture. Journal of Applied Mechanics, 68(1), 42- 48. https://doi.org/10.1115/1.1345525

Viguie, R., \& Kerschen, G. (2012). The nonlinear tuned vibration absorber. In MATEC Web of Conference (vol. 1), 05007. https://doi.org/10.1051/matecconf/20120105007

Volkova, V. E. (2013). Development of methods for nonparametric identification of models of mechanical systems. Procedia Engineering, 57, 1230-1235. https://doi.org/10.1016/j.proeng.2013.04.155

Volkova, V. E., \& Schneider, K. (2005). Qualitative theory and identification of dynamic system with one degree of freedom. International Applied Mechanics, 41(6), 704134-707139. https://doi.org/10.1007/s10778-005-0139-8 